\title{
Surface plasmons in metallic structures
}

\author{
J. M. Pitarke, ${ }^{1,2}$, V. M. Silkin ${ }^{2}$ E. V. Chulkov, ${ }^{2,3}$, and P. M. Echenique ${ }^{2,3}$ \\ ${ }^{1}$ Materia Kondentsatuaren Fisika Saila, Zientzi Fakultatea, Euskal Herriko Unibertsitatea, \\ 644 Posta kutxatila, E-48080 Bilbo, Basque Country, Spain \\ ${ }^{2}$ Donostia International Physics Center (DIPC) and Centro Mixto CSIC-UPV/EHU, \\ Manuel de Lardizabal Pasealekua, E-20018 Donostia, Basque Country, Spain \\ ${ }^{3}$ Materialen Fisika Saila, Kimika Fakultatea, Euskal Herriko Unibertsitatea, \\ 1072 Posta kutxatila, E-20080 Donostia, Basque Country, Spain
}

(Dated: September 5, 2018)

\begin{abstract}
Since the concept of a surface collective excitation was first introduced by Ritchie, surface plasmons have played a significant role in a variety of areas of fundamental and applied research, from surface dynamics to surface-plasmon microscopy, surface-plasmon resonance technology, and a wide range of photonic applications. Here we review the basic concepts underlying the existence of surface plasmons in metallic structures, and introduce a new low-energy surface collective excitation that has been recently predicted to exist.
\end{abstract}

PACS numbers: 71.45.Gm, 73.20.At, 78.47.+p, 78.68.+m

\section{INTRODUCTION}

The long-range nature of the Coulomb interaction between valence electrons in metals is known to yield collective behaviour, manifesting itself in the form of plasma oscillations. Pines and Bohm 1 were the first to suggest that the discrete energy losses experienced by fast electrons in passing through metals are due to the excitation of these plasma oscillations, the basic unit of energy being termed the plasmon: ${ }^{2} \hbar \omega_{p}=\hbar\left(4 \pi n e^{2} / m_{e}\right)^{1 / 2}$, where $n$ is the valence electron density and $m_{e}$ is the free electron mass $\frac{3}{2}$

Gabor ${ }^{4}$ investigated the excitation of plasma oscillations in thin foils, but assumed that the electric field is always zero at the surface. As a result, he did not find surface modes in the bounded plasma and reached the erroneus conclusion that the probability for plasma loss should decrease strongly with decreasing foil thickness. Ritchie ${ }^{\frac{5}{2}}$ was the first to find that the effect of the film boundaries is to cause the appearence of a new "lowered" loss at $\hbar \omega_{s}=\hbar \omega_{p} / \sqrt{2}$ due to the excitation of surface collective oscillations, the quanta of which Stern and Ferrell called the surface plasmon ${ }^{6}$

Ritchie's prediction of surface polarization causing lowenergy losses in metals was confirmed in a series of experiments carried out by Powell and Swan? ${ }^{7}$, who observed inelastic losses experienced by electrons scattered from newly evaporated layers of $\mathrm{Al}$ and $\mathrm{Mg}$. Since then, there has been a significant advance in both theoretical and experimental investigations of collective modes in the vacuum-solid interface.

The concept of the surface plasmon has played a key role in the understanding of fundamental properties of solids and in the interpretation of a large variety of experiments. For example, the classical image potential acting between a point classical charge and a metal surface was shown to be originated in the shifted zero point energy of the surface plasmon field, 8.9.10.11 the impact of the surfce plasmon on surface energies was addressed, the energy loss of charged particles moving outside a metal surface was shown to be due to the excitation of surface plasmons,$\frac{13,14}{1}$ and the centroid of the electron density induced by external potentials acting on a metal surface was demonstrated to be dictated by the wave-vector dependence of surface plasmons $\frac{15}{15}$ Explicit expressions for the surface-plasmon dispersion relied originally on simple models, such as the hydrodynamic, 16 specular-reflection, $\frac{17}{17}$ and infinite-barrier ${ }^{18}$ models. Accurate numerical calculations have also been performed from the knowledge of the eigenfunctions and eigenvalues of the Kohn-Sham hamiltonian of density-functional

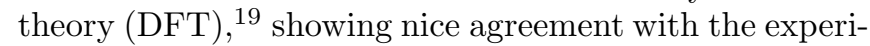
ments that have been carried out on clean, well characterized surfaces of the alkali metals ${ }^{20.21}$ These experiments also showed evidence for the existence of the so-called multipole surface plasmons that had been predicted by Benett 22

The long-wavelength ${ }^{23}$ surface-plasmon energy $\hbar \omega_{s}$ was derived by Ritchie in the nonrelativistic approximation, by assuming that the Coulomb interaction is instantaneous. However, if one is to describe the interaction of either relativistic electrons or light with solid surfaces, it is necessary to take into account the time needed for the propagation of the true retarded interaction. As a result of retardation, surface plasmons couple at wavelengths larger than $\sim 2 \pi c / \omega_{s}$ with the free electromagnetic field and yield what is now called a surfaceplasmon polariton ${ }^{24}$ At these large wavelengths, the surface-plasmon polariton exists over the entire frequency range from zero to an asymptotic value determined by the surface-plasmon frequency $\omega_{s}$. However, the corresponding dispersion curve never crosses the dispersion curve of free-space electromagnetic radiation. Hence, there is always a momentum mismatch between light and surface plasmons of the same frequency, so that light incident on an ideal surface cannot excite surface plasmons and, conversely, the surface plasmon cannot decay by emitting a photon. 
Teng and Stern $^{25}$ were the first to point out that any surface roughness permits the surface to impart some additional momentum to the surface-plasma oscillation, so that it can couple to electromagnetic radiation. Alternatively, prism coupling can be used to enhance the momentum of incident light, as demonstrated by Otto 26 and by Kretchmann and Raether ${ }^{27}$ Hence, surface plasmons have been employed in a wide spectrum of studies from electrochemistry, catalysis, wetting, thin organic condensates, and biosensing, ${ }^{28}$ to scanning tunneling microscopy,${ }^{29}$ the ejection of ions from surfaces, 30 surface dynamics, $\stackrel{31}{=}$ surface-plasmon microscopy $\stackrel{32}{,}$ and surface-plasmon resonance technology 33 Moreover, recent advances that allow metals to be structured and characterized on the nanometer scale have rekindled the long-standing interest in surface plasmons, one of the most attractive aspects of these collective excitations now being their use to concentrate light in subwavelength structures ${ }^{34}$ and to enhance transmission through periodic arrays of subwavelengths holes in optically thick metallic films, 35.36 as well as the possible fabrication of nanoscale photonic circuits operating at optical frequencies $\frac{37}{3}$

Since the typical energy of bulk and surface plasmons is of a few electronvolts, thermal excitation of these collective oscillations is improbable, so that the electronic properties near the Fermi level cannot be directly influenced by these excitations. Much more effective than ordinary bulk and surface plasmons in mediating, e.g., superconductivity would be the so-called acoustic plasmons with sound-like long-wavelength dispersion, 38 which have spurred over the years a remarkable interest and research activity 39 Acoustic plasma oscillations were observed in two-dimensionally confined and spatially separated multicomponent structures such as quantum wells and heterojunctions ${ }^{40.41}$ and were then proposed as possible candidates to mediate the attractive interaction leading to the formation of Cooper pairs in high- $T_{c}$ superconductors 42.43

Recently, it has been shown that metal surfaces where a partially occupied quasi two-dimensional (2D) surfacestate band coexists in the same region of space with the underlying three-dimensional (3D) continuum support a well-defined acoustic surface plasmon $\underline{44}$ This new lowenergy collective excitation exhibits linear dispersion at low wave vectors, and might therefore affect electron-hole (e-h) and phonon dynamics near the Fermi level $\frac{45}{1 t}$ has been demonstrated that it is a combination of the nonlocality of the 3D dynamical screening and the spill out of the $3 \mathrm{D}$ electron density into the vacuum which allows the formation of $2 \mathrm{D}$ electron-density acoustic oscillations at metal surfaces, since these oscillations would otherwise be completely screened by the surrounding $3 \mathrm{D}$ substrate ${ }^{46}$

In this paper, we first present an overview with the basic concepts underlying the existence of surface collective excitations in metallic structures, and we then introduce the new concept of acoustic surface plasmons. We begin in Section II with a brief discussion of the role that surface-plasmon excitation plays in the interaction of fast charged particles with solid surfaces, since it is precisely the investigation of electron energy loss in thin foils which brought Ritchie to the realization that surface collective excitations exist at the lowered frequency $\omega_{s}, \underline{\underline{5}}$ The detection of surface plasmons and their dispersion is discussed in Section III, in the framework of angle-resolved inelastic electron scattering experiments. Localized surface plasmons and the use of sum rules that provide insight of surface-plasmon energies in metallic structures of arbitrary geometry are introduced in Section IV. Section V is devoted to the retarded region, where surface plasmons couple with the free electromagnetic field. Acoustic surface plasmons are introduced in Section VI.

Unless stated otherwise, atomic units are used throughout, i.e., $e^{2}=\hbar=m_{e}=1$.

\section{PLASMA LOSSES BY FAST CHARGED PARTICLES IN SOLIDS}

Let us consider a recoiless fast point particle of charge $Z_{1}$ moving in an arbitrary inhomogeneous many-electron system with nonrelativistic velocity $\mathbf{v}$, for which retardation effects and radiation losses can be neglected $\underline{47}$ The charge density of the probe particle is simply a delta function of the form

$$
\rho^{e x t}(\mathbf{r}, t)=Z_{1} \delta\left(\mathbf{r}-\mathbf{r}_{0}-\mathbf{v} t\right)
$$

and the energy that this classical particle loses per unit time due to electronic excitations in the medium can be written as $\underline{48}$

$$
-\frac{d E}{d t}=-\int d \mathbf{r} \rho^{e x t}(\mathbf{r}, t) \frac{\partial V^{i n d}(\mathbf{r}, t)}{\partial t},
$$

where $V^{i n d}(\mathbf{r}, t)$ is the potential induced by the probe particle at position $\mathbf{r}$ and time $t$.

To first order in the external perturbation, timedependent perturbation theory yields

$$
\begin{aligned}
V^{i n d}(\mathbf{r}, t) & =\int d \mathbf{r}^{\prime} \int_{-\infty}^{+\infty} d t^{\prime} \int_{-\infty}^{+\infty} \frac{d \omega}{2 \pi} \mathrm{e}^{-i \omega\left(t-t^{\prime}\right)} \\
& \times \tilde{W}\left(\mathbf{r}, \mathbf{r}^{\prime} ; \omega\right) \rho^{e x t}\left(\mathbf{r}^{\prime}, t^{\prime}\right)
\end{aligned}
$$

where

$$
\tilde{W}\left(\mathbf{r}, \mathbf{r}^{\prime} ; \omega\right)=W\left(\mathbf{r}, \mathbf{r}^{\prime} ; \omega\right)-v\left(\mathbf{r}, \mathbf{r}^{\prime}\right),
$$

$v\left(\mathbf{r}, \mathbf{r}^{\prime}\right)$ being the bare Coulomb interaction and $W\left(\mathbf{r}, \mathbf{r}^{\prime} ; \omega\right)$ being the so-called screened interaction, which is usually expresed in terms of the density-response function of the many-electron system 49

Eq. (3) is a general expression for the energy loss of a classical particle moving in an arbitrary inhomogeneous electron system that is characterized by the screened interaction $W\left(\mathbf{r}, \mathbf{r}^{\prime} ; \omega\right)$. Here we consider a solid target consisting of a fixed uniform positive background (jellium) 
plus a neutralizing cloud of interacting valence electrons, which will be described by either an infinite or a planebounded electron gas.

\section{A. Infinite electron gas}

In the case of an infinite homogeneous electron gas that is translationally invariant in all directions, Eqs. (11)-(3) yield the following expression for the so-called stopping power, i.e., the energy that the probe particle loses per unit path length:

$$
-\frac{d E}{d x}=-Z_{1}^{2} \int \frac{d^{3} \mathbf{k}}{(2 \pi)^{3}} \mathbf{k} \cdot \mathbf{v} \operatorname{Im} \tilde{W}(k, \mathbf{k} \cdot \mathbf{v}),
$$

$\tilde{W}(k, \omega)$ being the 3D Fourier transform of $\tilde{W}\left(\mathbf{r}, \mathbf{r}^{\prime} ; \omega\right)$, which is typically expressed in the form

$$
\tilde{W}(k, \omega)=\frac{4 \pi}{k^{2}}\left[\epsilon^{-1}(k, \omega)-1\right],
$$

where $\epsilon^{-1}(k, \omega)$ is the so-called inverse dielectric function of the electron gas.

At high projectile velocities $\left(v_{F}<<v, v_{F}\right.$ being the Fermi velocity), the zero-point motion of the electron gas can be neglected and it can be considered, therefore, as if it were at rest. In this approximation, the dielectric function $\epsilon(k, \omega)$ of a homogeneous electron gas takes the form ${ }^{50}$

$$
\epsilon(k, \omega)=1-\frac{\omega_{p}^{2}}{\omega(\omega+i \eta)-k^{4} / 4},
$$

which at long wavelengths $(k \rightarrow 0)$ yields the classical Drude dielectric function

$$
\epsilon(\omega)=1-\frac{\omega_{p}^{2}}{\omega(\omega+i \eta)},
$$

$\omega_{p}$ being the bulk plasma frequency and $\eta$ a positive infinitesimal.

The dielectric function of Eq. (7) describes both collective and single-particle excitations. At wave vectors $\mathbf{k}$ smaller than a cut-off wave vector of magnitude $k_{c} \sim \omega_{p} / v_{F}$, the many-electron system can be expected to behave collectively, losses being dominated by the excitation of plasma oscillations ${ }^{2}$ At wave vectors of magnitude larger than $k_{c}$, losses are dominated by the excitation of electron-hole (e-h) pairs. Using Eq. (7) and assuming that $v>>v_{F}$, Eq. (5) yields

$$
-\frac{d E}{d x}=Z_{1}^{2} \frac{\omega_{p}^{2}}{v^{2}}\left[\ln \frac{k_{c}}{\omega_{p} / v}+\ln \frac{2 v}{k_{c}}\right]
$$

or, equivalently,

$$
-\frac{d E}{d x}=Z_{1}^{2} \frac{\omega_{p}^{2}}{v^{2}} \ln \frac{2 v^{2}}{\omega_{p}} .
$$

The first term of Eq. (9), which can also be obtained by using the Drude dielectric function of Eq. (8), represents the contribution to the stopping power from losses to collective excitations at wave vectors of magnitude smaller than $k_{c}$. The second term of Eq. (9) represents the contribution from losses to single-particle excitations at wave vectors above $k_{c}$.

\section{B. Plane-bounded electron gas}

In the case of a plane-bounded electron gas that is translationally invariant in two directions, which we take to be normal to the $z$ axis, Eqs. (11)-(3) yield the following expression for the energy that the probe particle loses per unit time:

$$
\begin{aligned}
-\frac{d E}{d t} & =i \frac{Z_{1}^{2}}{\pi} \int \frac{d^{2} \mathbf{q}}{(2 \pi)^{2}} \int_{-\infty}^{+\infty} d t^{\prime} \int_{0}^{\infty} d \omega \omega \\
& \times \mathrm{e}^{-i\left(\omega-\mathbf{q} \cdot \mathbf{v}_{\|}\right)\left(t-t^{\prime}\right)} \tilde{W}\left[z(t), z\left(t^{\prime}\right) ; q, \omega\right],
\end{aligned}
$$

where $\mathbf{q}$ is a $2 \mathrm{D}$ wave vector in the plane of the surface, $\mathbf{v}_{\|}$represents the component of the velocity that is parallel to the surface, $z(t)$ represents the position of the projectile relative to the surface, and $\tilde{W}\left(z, z^{\prime} ; q, \omega\right)$ is the 2D Fourier transform of $\tilde{W}\left(\mathbf{r}, \mathbf{r}^{\prime} ; \omega\right)$.

In the simplest possible model of a bounded semiinfinite electron gas, one characterizes the electron gas at $z \geq 0$ by a local dielectric function which jumps discontinuosly at the surface from unity outside $(z<0)$ to $\epsilon(\omega)$ inside $(z>0)$. By imposing the ordinary boundary conditions of continuity of the potential and the normal component of the displacement vector at $z=0$, one finds

$$
\tilde{W}\left(z, z^{\prime} ; \mathbf{q}, \omega\right)=\frac{2 \pi}{q} \begin{cases}-g(\omega) \mathrm{e}^{-q\left(|z|+\left|z^{\prime}\right|\right)} & z<0 \\ {\left[\epsilon^{-1}(\omega)-1\right] \mathrm{e}^{-q\left|z-z^{\prime}\right|}+\epsilon^{-1}(\omega) g(\omega) \mathrm{e}^{-q\left(|z|+\left|z^{\prime}\right|\right)},} & z>0\end{cases}
$$

where

$$
g(\omega)=\frac{\epsilon(\omega)-1}{\epsilon(\omega)+1}
$$

is the long-wavelength $(q \rightarrow 0)$ limit of the so-called 
surface-response function 51 In this limit, the dielectric function $\epsilon(\omega)$ takes the Drude form dictated by Eq. (8).

In the following, we shall explicitly consider particle trajectories that are normal and parallel to the surface.

\section{Normal trajectory}

Let us consider a situation in which the probe particle moves along a normal trajectory from the vacuum side of the surface $(z<0)$ and enters the solid at $z=t=0$. The position of the projectile relative to the surface is then $z(t)=v t$. Assuming that the electron gas at $z \geq 0$ can be described by the Drude dielectric function of Eq. (8) and introducing Eq. (12) into Eq. (11), one finds the following expression for the energy that the probe particle loses per unit path length:

$$
-\frac{d E}{d z}=\frac{Z_{1}^{2}}{v^{2}} \begin{cases}\omega_{s}^{2} f\left(2 \omega_{s}|z| / v\right), & z<0 \\ \omega_{p}^{2}\left[\ln \left(k_{c} v / \omega_{p}\right)-h\left(\omega_{p} z / v\right)\right]+\omega_{s}^{2} h\left(\omega_{s} z / v\right), & z>0\end{cases}
$$

where

$$
h(\alpha)=2 \cos (\alpha) f(\alpha)-f(2 \alpha),
$$

with $f(\alpha)$ being given by the following expression:

$$
f(\alpha)=\int_{0}^{\infty} \frac{x \mathrm{e}^{-\alpha x}}{1+x^{2}}
$$

The Drude dielectric function of Eq. (8), which assumes that infinitely long-lived plasmons at a singlefrequency $\omega_{p}$ are the only possible bulk excitations, is only sustainable at wave vectors below a cut-off $k_{c}$. Hence, this cut-off has been introduced into the bulk (logarithmic) term of Eq. (14), which yields a contribution to the energy loss that coincides with the plasmon contribution of Eq. (9). Contributions to the energy loss that are due to the excitation of e-h pairs are not included in Eq. (14).

In the absence of the boundary at $z=0$, the positiondependent $f(\alpha)$ and $h(\alpha)$ terms entering Eq. (14) would not be present, and the energy loss would be that of charged particles moving in an infinite plasma. When the probe particle is moving outside the solid, the effect of the boundary is to cause energy loss at the lowered plasma frequency $\omega_{s}$. When the probe particle is moving inside the solid, the effect of the boundary is to cause both a decrease in loss at the bulk plasma frequency $\omega_{p}$ and an additional loss at the lowered plasma frequency $\omega_{s}$, as predicted by Ritchie. ${ }^{5}$

Now we consider the real situation in which a fast charged particle passes through a finite foil of thickness $a$ (see Fig. 1). Assuming that the foil is thick enough for the effect of each boundary to be the same as in the case of a semi-infinite medium, and integrating along the whole trajectory from minus to plus infinity, one finds the total energy that the probe particle loses to collective excitations:

$$
-\Delta E=\frac{Z_{1}^{2}}{v^{2}}\left[a \omega_{p}^{2} \ln \frac{k_{c} v}{\omega_{p}}-\frac{\pi}{2} \omega_{p}+\pi \omega_{s}\right] .
$$

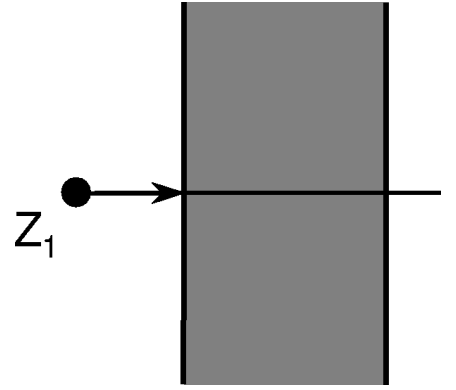

FIG. 1: Particle of charge $Z_{1}$ passing through a finite foil of thickness $a$.

This is the result first derived by Ritchie in a different way,, 5 wich brought him to the realization that surface collective excitations exist at the lowered frequency $\omega_{s}$. The first term of Eq. (17), which is proportional to the thickness of the film represents the bulk contribution, which would also be present in the absence of the boundaries. The second and third terms, which are both due to the presence of the boundaries and become more important as the foil thickness decreases, represent the decrease in the energy loss at the plasma frequency $\omega_{p}$ and the energy loss at the lowered frequency $\omega_{s}$, respectively. Eq. (17) also shows that the net boundary effect is an increase in the total energy loss above the value which would exist in its absence, as noted by Ritchie.$^{\frac{5}{}}$

Ritchie also considered the coupling that exists between the two surfaces for finite values of the film thickness $a$. He found the following dispersion relation between the frequency of surface-plasma oscillations and the wave number $q$ :

$$
\omega=\omega_{s}\left[1 \pm \mathrm{e}^{-a q}\right]^{1 / 2}
$$

the exponential factor being a consequence of the interaction between the two surfaces. This equation has two 


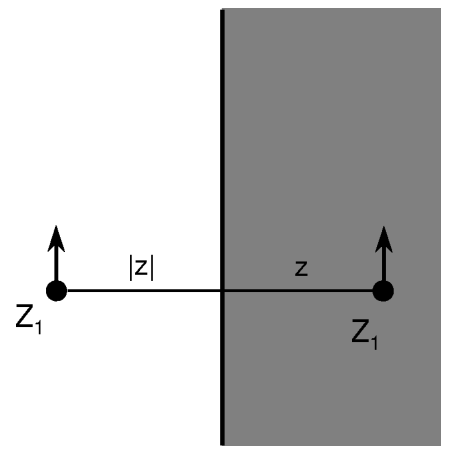

FIG. 2: Particle of charge $Z_{1}$ moving at a fixed distance $z$ from the surface of a plane-bounded electron gas.

limiting cases, as discussed by Ferrell ${ }^{52}$ At short wavelengths $(q a>>1)$, the surface waves become decoupled and each surface sustains independent oscillations at the reduced frequency $\omega_{s}$ characteristic of a semi-infinite electron gas with a single plane boundary. At long wavelengths $(q a<<1)$, there are "normal" oscillations at $\omega_{p}$ and "tangential" $2 \mathrm{D}$ oscillations at

$$
\omega_{2 D}=(2 \pi n a q)^{1 / 2},
$$

which were later discussed by Stern ${ }^{53}$ and observed in artificially structured semiconductors 54 and more recently in a metallic surface-state band on a silicon surface ${ }^{55}$

\section{Parallel trajectory}

Now we restrict our attention to the case of charged particles moving with constant velocity at a fixed distance $z$ from the surface (see Fig. 22). Eq. (11) then yields

$$
\begin{aligned}
-\frac{d E}{d x}= & -\frac{2}{v} Z_{1}^{2} \int \frac{d^{2} \mathbf{q}}{(2 \pi)^{2}} \int_{0}^{\infty} d \omega \omega \\
& \times \operatorname{Im} \tilde{W}(z, z ; \mathbf{q}, \omega) \delta(\omega-\mathbf{q} \cdot \mathbf{v}) .
\end{aligned}
$$

Assuming that the electron gas at $z \geq 0$ can be described by the Drude dielectric function of Eq. [8] and introducing Eq. (12) into Eq. (20), one finds

$$
-\frac{d E}{d x}=\frac{Z_{1}^{2}}{v^{2}} \begin{cases}\omega_{s}^{2} K_{0}\left(2 \omega_{s}|z| / v\right), & z<0 \\ \omega_{p}^{2}\left[\ln \left(k_{c} v / \omega_{p}\right)-K_{0}\left(2 \omega_{p} z / v\right)\right]+\omega_{s}^{2} K_{0}\left(2 \omega_{s} z / v\right), & z>0,\end{cases}
$$

where $K_{0}(\alpha)$ is the zero-order modified Bessel function ${ }^{56}$

For particle trajectories outside the solid $(z<0)$, Eq. (21) reproduces the classical expression of Echenique and Pendry $\stackrel{13}{\underline{13}}$ For particle trajectories inside the solid $(z>0)$, Eq. (21) reproduces the result first obtained by Nuñez et al. ${ }^{57}$ As in the case of a normal trajectory, when the particle moves inside the solid the effect of the boundary is to cause a decrease in loss at the bulk plasma frequency $\omega_{p}$ and an additional loss at the lowered plasma frequency $\omega_{s}$.

\section{INELASTIC ELECTRON SCATTERING}

The most commonly used experimental arrangement for detecting surface plasmons by the fields of moving charged particles is based on angle-resolved inelastic electron scattering 58.59 Fig. 3 shows a schematic drawing of the scattering geometry. A monochromatic beam of electrons, incident on a flat surface at an angle $\theta_{i}$, is back scattered and detected by an angle-resolved energy analyzer positioned at an angle $\theta_{f}$. Inelastic events can occur, either before or after the elastic event, by exciting a surface mode of frequency $\omega(q)$. The energy and lifetime of this mode are determined by the corresponding energy-loss peak in the spectra, and the momentum $q$ parallel to the surface is obtained from the measured angles $\theta_{i}$ and $\theta_{f}$.

The inelastic-scattering cross section corresponding to a process in which an electronic excitation of energy $\omega$ and parallel momentum $q$ is created at the surface of a semi-infinite electron gas is dictated by the imaginary part of the surface-response function $g(q, \omega), 51$ which in the long-wavelength limit is given by Eq. (13). In a freeelectron gas (jellium), the dielectric function $\epsilon(\omega)$ entering Eq. (13) is the Drude dielectric function of Eq. (8). Hence, in a gas of free electrons $\operatorname{Im} g(q, \omega)$ becomes a delta function peaked at the surface-plasmon energy $\omega_{s}$.

The classical picture leading to Eqs. (8) and (13), which is correct only in the long-wavelength limit, ignores both the nonlocality of the electronic response of the system and the microscopic spatial distribution of the electron density near the surface. Feibelman showed that up to first order in an expansion in powers of $q$, the surface-response function of a jellium surface can be 


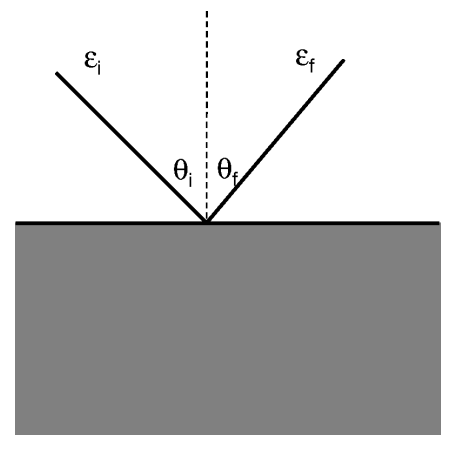

FIG. 3: Schematic drawing of the scattering geometry in angle-resolved inelastic electron scattering experiments. By exciting a surface mode of frequency $\omega(q)$, the energy of detected electrons becomes $\varepsilon_{f}=\varepsilon_{i}-\omega(q)$. The momentum $q$ is determined from $q=\sqrt{2}\left[\sqrt{\varepsilon_{i}} \sin \theta_{i}-\sqrt{\varepsilon_{f}} \sin \theta_{f}\right]$

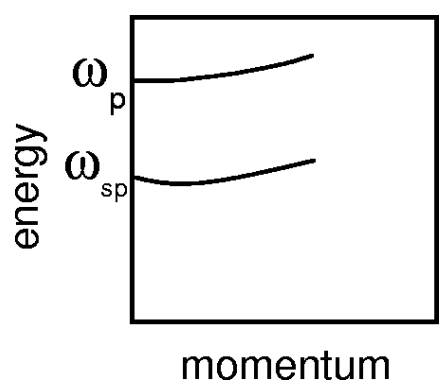

FIG. 4: Schematic drawing of the bulk and surface plasmon energy dispersions in typical metal surfaces. While in the case of bulk plasmons the initial slope is usually (but not always) positive, the surface-plasmon energy dispersion is negative for typical metal surfaces.

written as 15

$$
g(q, \omega)=\frac{\epsilon(\omega)-1}{\epsilon(\omega)+1}\left[1+2 q d_{\perp}(\omega) \frac{\epsilon(\omega)}{\epsilon(\omega)+1}\right]+O\left(q^{2}\right),
$$

where $d_{\perp}(\omega)$ represents the centroid of the induced electron density. Self-consistent density-functional calculations of $g(q, \omega)$ and $d_{\perp}(\omega)$ have demonstrated that in the case of jellium surfaces in the range of typical bulk densities the surface-plasmon energy $\omega(q)$ [where $\operatorname{Im} g(q, \omega)$ is maximum] is at nonvanishing but small $\mathbf{q}$ wave vectors lower than $\omega_{s}$, in agreement with experiment ${ }^{20,21}$ This is illustrated in Fig. 4 where the surface-plasmon energy dispersion is drawn schematically, showing that the initial slope is negative. For an interpretation of negative dispersion and comparison to experiment see Ref. 51.

At jellium surfaces, the actual surface-response function reduces in the long-wavelength limit to Eq. (13) with the Drude $\epsilon(\omega)$ of Eq. (8), and surface plasmons are therefore expected to be infinitely long-lived excitations. However, energy-loss measurements at simple metal surfaces indicate that surface plasmons exhibit a finite width even at $q=0.60$ Since surface plasmons are dictated in this $q=0$ limit by bulk properties through
TABLE I: Relative widths $\Delta \omega_{s} / \omega_{s}$ of surface plasmons, as derived from the imaginary part of the surface-response function of Eq. (13) with measured values of the bulk dielectric function $\epsilon(\omega)$ (theory $)^{51}$ and from the surface-loss measurements at $q=0$ (experiment) ${ }^{60}$

\begin{tabular}{lcccccc}
\hline \hline & $\mathrm{Ag}$ & $\mathrm{K}$ & $\mathrm{Al}$ & $\mathrm{Mg}$ & $\mathrm{Hg}$ & $\mathrm{Li}$ \\
\hline Theory & 0.027 & 0.035 & 0.035 & 0.16 & 0.18 & 0.33 \\
Experiment & 0.027 & 0.1 & 0.24 & 0.16 & 0.16 & 0.35 \\
\hline \hline
\end{tabular}
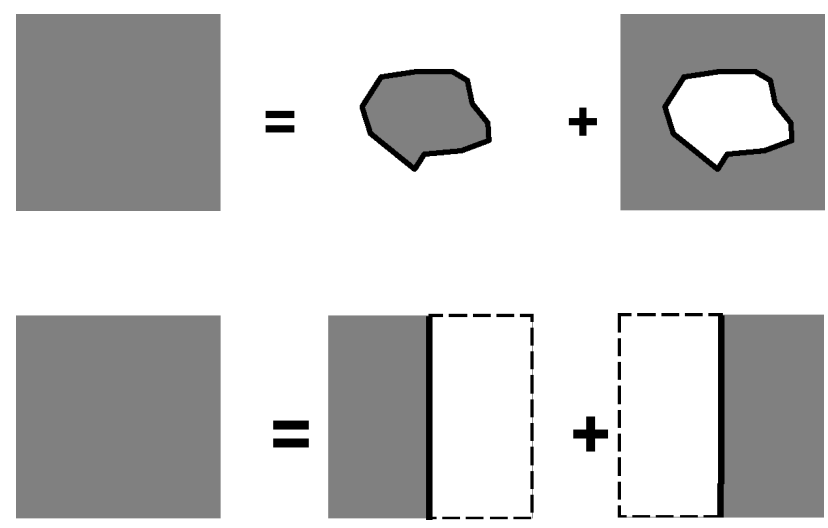

FIG. 5: Complementary systems in which the regions of plasma and vacuum are interchanged. The top panel represents the general situation. The bottom panel represents a half-space filled with metal and interfaced with vacuum. The surface-mode frequencies $\omega_{s_{1}}$ and $\omega_{s_{2}}$ of these systems fulfill the sum rule of Eq. (23).

the dielectric function $\epsilon(\omega)$, the experimental surfaceplasmon widths $\Delta \omega_{s}$ at $q=0$ should be approximately described by using in Eq. (13) the measured bulk dielectric function $\epsilon(\omega)$ instead of its Drude counterpart. Table \ exhibits the relative widths $\Delta \omega_{s} / \omega_{s}$ of surface plasmons derived in this way, 51 together with surfaceloss measurements at $q=0.60$ Although in the case of $\mathrm{Ag}, \mathrm{Li}, \mathrm{Hg}$, and $\mathrm{Mg}$ the surface-plasmon width is well described by introducing the measured bulk dielectric function into Eq. (13), the surface-plasmon widths of $\mathrm{K}$ and $\mathrm{Al}$ are considerably larger than predicted in this simple way. This shows that an understanding of surfaceplasmon broadening mechanisms requires a careful analysis of the actual band structure of the solid. Approximate treatments of the impact of the band structure on the surface-plasmon energy dispersion have been developed by several authors,$\underset{61.62 .63 .64 .65}{ }$ but a first-principles description of surface energy-loss measurements has not been carried out yet.

\section{SUM RULES}

Sum rules have played a key role in providing insight in the investigation of a variety of physical situations. A 
useful sum rule for the surface modes in complementary media with arbitrary geometry was introduced by Apell et al. $\frac{66}{6}$ which in the special case of a metal/vacuum interface implies that $\underline{67}$

$$
\omega_{s_{1}}^{2}+\omega_{s_{2}}^{2}=\omega_{p}^{2}
$$

where $\omega_{s_{1}}$ is the surface-mode frequency of our system, and $\omega_{s_{2}}$ represents the surface mode of a second complementary system in which the regions of plasma and vacuum are interchanged (see Fig. 沾).

For example, a half-space filled with a metal of bulk plasma frequency $\omega_{p}$ and interfaced with vacuum maps into itself (see Fig. 5), and therefore Eq. (23) yields

$$
\omega_{s_{1}}=\omega_{s_{2}}=\omega_{p} / \sqrt{2}
$$

which is the frequency of plasma oscillations at a metal/vacuum planar interface.

Other examples are a metal sphere in vacuum, which sustains localized Mie plasmons at frequencies

$$
\omega_{l}=\omega_{p} \sqrt{\frac{l}{2 l+1}},
$$

with $l=1,2, \ldots$, and a spherical void in a metal, which shows Mie plasmons at frequencies

$$
\omega_{l}=\omega_{p} \sqrt{\frac{l+1}{2 l+1}} .
$$

The squared surface-mode frequencies of the sphere [Eq. (25)] and the void [(Eq. (26)] add up to $\omega_{p}^{2}$ for all $l$, as required by Eq. (23).

Now we consider a situation in which there are two interfaces, as occurs in the case of a thin film and approximately occurs in the case of multishell fullerenes 68 and carbon nanotubes ${ }^{69}$ Apell et al ${ }^{66}$ have proved a second sum rule, which relates the surface modes corresponding to the in-phase and out-of-phase linear combinations of the screening charge densities at the interfaces. In the case of metal/vacuum interfaces this sum rule takes the form of Eq. (23), but now $\omega_{s_{1}}$ and $\omega_{s_{2}}$ being in-phase and out-of-phase modes of the same system.

For a Drude metal film with equal and abrupt planar surfaces, the actual values of $\omega_{s_{1}}$ and $\omega_{s_{2}}$ are those given by Eq. (18). For a spherical fullerene molecule described by assigning a Drude dielectric function to every point between the inner and outer surfaces of radii $r_{1}$ and $r_{2}$ (see Fig. 6), one finds the following frequencies for the in-phase and out-of-phase surface modes ${ }^{70}$

$$
\omega_{s}^{2}=\frac{\omega_{p}^{2}}{2}\left[1 \pm \frac{1}{2 l+1} \sqrt{1+4 l(l+1)\left(r_{1} / r_{2}\right)^{2 l+1}}\right]
$$

which fulfill the sum rule dictated by Eq. (23).

\section{SURFACE-PLASMON POLARITONS}

Planar surface plasmons are known to be traced to the peaks of the imaginary part of the surface response

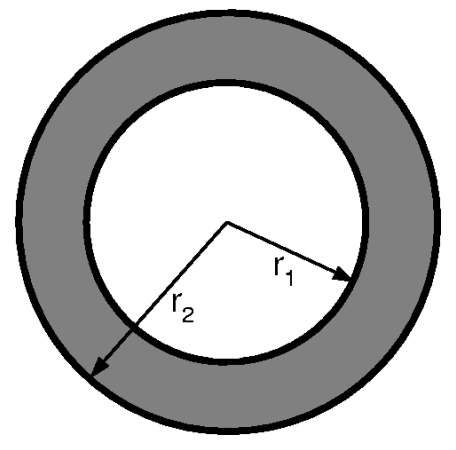

FIG. 6: Fullerene molecule of inner and outer radii $r_{1}$ and $r_{2}$. A Drude dielectric function is assigned to every point between the inner and outer surfaces.

function $g(q, \omega), 51$ which in the long-wavelength limit is given by Eq. (13). This equation yields the classical nondispersive surface-plasmon frequency $\omega_{s}$ given by

$$
\epsilon\left(\omega_{s}\right)+1=0,
$$

which in the case of a semi-infinite Drude metal [see Eq. [8] ] is $\omega_{s}=\omega_{p} / \sqrt{2}$.

The long-wavelength surface-plasmon condition of Eq. (28) has been derived in the nonrelativistic approximation, by neglecting retardation of the Coulomb interaction. Hence, Eq. (28) yields a good representation of surface plasma oscillations only at wavelengths that are large compared to the Fermi wavelength $\left(q<<k_{F} \sim\right.$ $1 \AA^{-1}, k_{F}$ being the Fermi momentum) but small compared to the wavelength of light at optical frequencies $\left(q>>\omega_{s} / c \sim 0.005 \AA^{-1}\right)$. In a typical inelastic electron scattering experiment, however, the finite angular acceptance of the energy-loss spectrometer garantees that the momentum transfer $q$ be larger than $\omega_{s} / c$, so that the retarded region of the surface plasmon dispersion is not observed ${ }^{71}$

Considering the full set of Maxwell equations and still assuming that the wavelength is long enough for a classical description of the metal/vacuum interface to be justified $\left(q<<k_{F}\right)$, one finds that due to retardation the surface-plasmon condition of Eq. (28) must be replaced by ${ }^{71}$

$$
\frac{\epsilon\left(\omega_{s}\right)}{\kappa\left(\omega_{s}\right)}+\frac{1}{\kappa^{\prime}\left(\omega_{s}\right)}=0
$$

where

$$
\kappa(\omega)=\sqrt{q^{2}-\epsilon(\omega) \frac{\omega^{2}}{c^{2}}}
$$

and

$$
\kappa^{\prime}(\omega)=\sqrt{q^{2}-\frac{\omega^{2}}{c^{2}}} .
$$

In the case of a semi-infinite Drude metal, Eqs. (29)-(31) yield the surface-plasmon dispersion

$$
\omega^{2}(q)=\omega_{p}^{2} / 2+c^{2} q^{2}-\sqrt{\omega_{p}^{4} / 4+c^{4} q^{4}},
$$




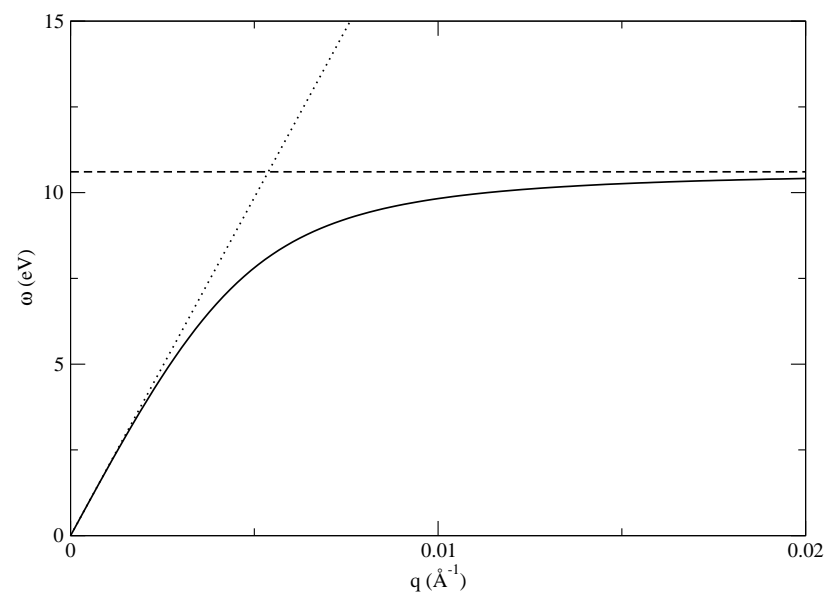

FIG. 7: The solid line represents the dispersion of surfaceplasmon polaritons on a semi-infinite Drude metal with $\omega_{p}=$ $15 \mathrm{eV}$, as obtained from Eq. (32). In the retarded region $\left(q<\omega_{p} / c\right)$, the surface-plasmon polariton dispersion curve approaches the light line $\omega=c q$ (dotted line). At short wavelengths $(q>>\omega / c)$, the dispersion curve approaches asymptotically the non-retarded surface-plasmon frequency $\omega_{s}=\omega_{p} / \sqrt{2}$ (dashed line).

or, equivalently,

$$
q(\omega)=\frac{\omega}{c} \sqrt{\frac{\omega^{2}-\omega_{p}^{2}}{2 \omega^{2}-\omega_{p}^{2}}},
$$

which we have represented in Fig. 7 by a solid line, together with the non-retarded surface-plasmon frequency $\omega_{s}$ (dashed line) and the light line $\omega=c q$ (dotted line). In the retarded region, where $q<\omega_{s} / c$, surface plasmons couple with the free electromagnetic field, thereby becoming what is called a surface-plasmon polariton. In the non-retarded limit $\left(q>>\omega_{s} / c\right)$, one finds the nondispersive surface-plasmon frequency $\omega_{s}$.

Significant deviations from the classical surfaceplasmon dispersion of Eq. (32) (like the negative dispersion drawn in Fig. (4), which are typically observed in electron scattering experiments, are only present at wave vectors larger than those considered in Fig. 7 At $\mathbf{q}$ wave vectors in the range $\omega_{s} / c<<q<<k_{F}$, the surface plasmon does not disperse.

\section{ACOUSTIC SURFACE PLASMONS}

A variety of metal surfaces, such as $\mathrm{Be}(0001)$ and the (111) surfaces of the noble metals $\mathrm{Cu}, \mathrm{Ag}$, and $\mathrm{Au}$, are known to support a partially occupied band of Shockley surface states with energies near the Fermi level (see Fig. 8) ${ }^{72}$ Since these states are strongly localized near the surface and disperse with momentum parallel to the surface, they can be considered to form a quasi $2 \mathrm{D}$ surface-state band with a 2D Fermi energy equal to the surface-state binding energy at the $\bar{\Gamma}$ point (see Table 【I).

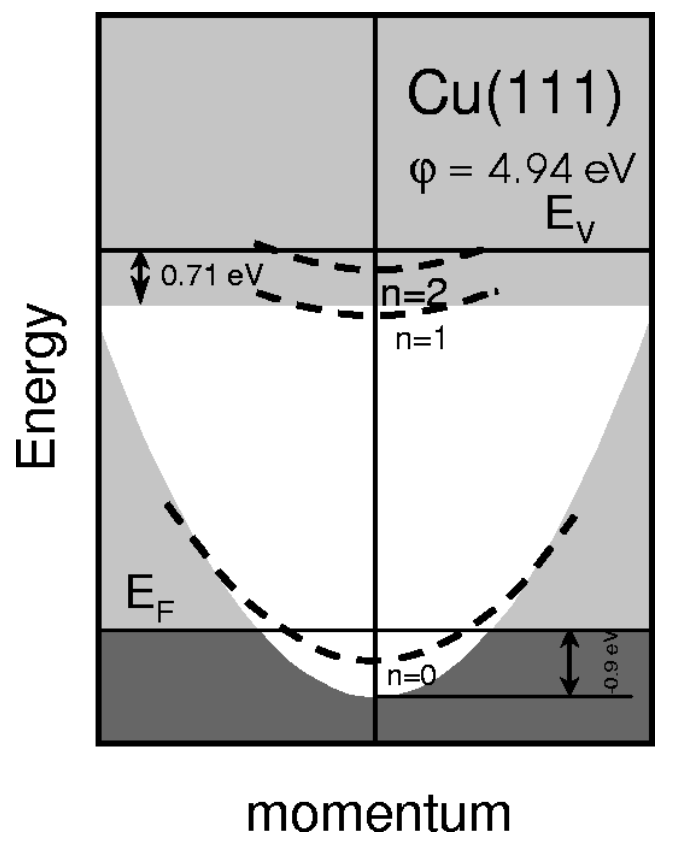

FIG. 8: Schematic representation of the surface band structure on $\mathrm{Cu}(111)$ near the $\bar{\Gamma}$ point. The shaded region represents the projection of the bulk bands.

TABLE II: Binding energies $\left(\varepsilon_{F}^{2 D}\right)$ of surface states at the $\bar{\Gamma}$ point of $\mathrm{Be}(0001)$ and the (111) surfaces of the noble metals $\mathrm{Cu}, \mathrm{Ag}$, and $\mathrm{Au} . v_{F}^{2 D}$ and $m^{2 D}$ represent the corresponding $2 \mathrm{D}$ Fermi velocity and effective mass, respectively. $v_{F}^{2 D}$ is expressed in units of the Bohr velocity $v_{0}=e^{2} / \hbar$.

\begin{tabular}{lccc}
\hline \hline & $\varepsilon_{F}^{2 D}(\mathrm{eV})$ & $v_{F}^{2 D} / v_{0}$ & $m^{2 D}$ \\
\hline $\mathrm{Be}(0001)$ & 2.8 & 0.41 & 1.18 \\
$\mathrm{Cu}(111)$ & 0.44 & 0.28 & 0.42 \\
$\mathrm{Ag}(111)$ & 0.065 & 0.11 & 0.44 \\
$\mathrm{Au}(111)$ & 0.48 & 0.35 & 0.28 \\
\hline \hline
\end{tabular}

In the absence of the 3D substrate, Shockley surface states would support a $2 \mathrm{D}$ collective oscillation, the energy of this plasmon being given by Eq. (19) with na replaced by the $2 \mathrm{D}$ density of occupied surface states: $n^{2 D}=\varepsilon_{F}^{2 D} / \pi$. Eq. (19) shows that at very long wavelengths plasmons in a 2D electron gas have low energies; however, they do not affect e-h and phonon dynamics near the Fermi level, due to their square-root dependence on the wave vector. Much more effective than ordinary 2D plasmons in mediating, e.g., superconductivity would be the so-called acoustic plasmons with sound-like longwavelength dispersion.

Here we show that in the presence of the 3D substrate the dynamical screening at the surface provides a mechanism for the existence of a new acoustic collective mode, whose energy exhibits a linear dependence on the 2D wave number. 


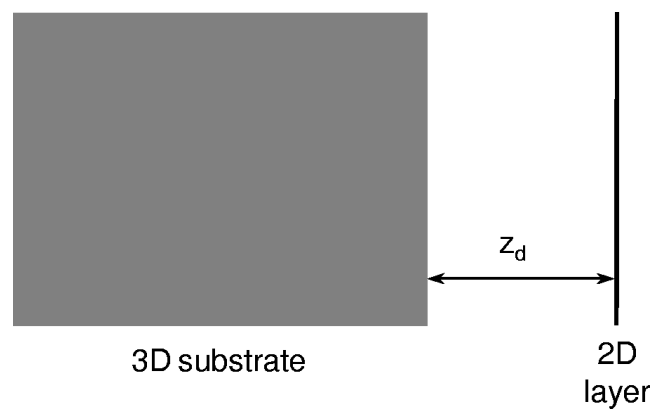

FIG. 9: Surface-state electrons comprise a 2D sheet of interacting free electrons at $z=z_{d}$. All other states of the semi-infinite metal comprise a plane-bounded 3D electron gas at $z \leq 0$. The metal surface is located at $z=0$.

\section{A. A simple model}

First of all, we consider a simplified model in which surface-state electrons comprise a 2D electron gas at $z=$ $z_{d}$ (see Fig. 9), while all other states of the semi-infinite metal comprise a 3D substrate at $z \leq 0$ represented by the Drude dielectric function of Eq. (8). Within this model, one finds that both e-h and collective excitations occurring within the $2 \mathrm{D}$ gas can be described with the use of an effective 2D dielectric function, which in the random-phase approximation (RPA) takes the form 46

$$
\epsilon_{e f f}^{2 D}(q, \omega)=1-\left[\frac{2 \pi}{q}+\tilde{W}\left(z_{d}, z_{d} ; q, \omega\right)\right] \chi_{2 D}^{0}(q, \omega)
$$

$\tilde{W}\left(z, z^{\prime} ; q, \omega\right)$ being the screened interaction of Eq. (12), and $\chi_{2 D}^{0}(q, \omega)$ being the noninteracting density-response function of a $2 \mathrm{D}$ electron gas 53

In the absence of the $3 \mathrm{D}$ substrate, $\tilde{W}\left(z, z^{\prime} ; q, \omega\right)$ is simply zero and $\epsilon_{e f f}^{2 D}(q, \omega)$ coincides, therefore, with the RPA dielectric function of a $2 \mathrm{D}$ electron gas, which in the long-wavelength $(q \rightarrow 0)$ limit has one single zero corresponding to collective excitations at $\omega=\omega_{2 D}$.

In the presence of a 3D substrate that is spatially separated from the $2 \mathrm{D}$ sheet $\left(z_{d}>0\right)$, the long-wavelength limit of $\epsilon_{e f f}^{2 D}(q, \omega)$ has two zeros. One zero corresponds to a high-frequency oscillation of energy $\omega^{2}=\omega_{s}^{2}+\omega_{2 D}^{2}$ in which $2 \mathrm{D}$ and $3 \mathrm{D}$ electrons oscillate in phase with one another. The other zero corresponds to a low-frequency acoustic oscillation in which both $2 \mathrm{D}$ and $3 \mathrm{D}$ electrons oscillate out of phase. The energy of this low-frequency mode is found to be of the form ${ }^{46}$

$$
\omega=\alpha v_{F}^{2 D} q
$$

with $\alpha>1$. For small values of the $z_{d}$ coordinate $\left(z_{d}<<\right.$ 1), $\alpha \rightarrow 1$ and the sound velocity approaches, therefore, the Fermi velocity of the $2 \mathrm{D}$ sheet. For $z_{d}>>1$, the noninteracting $2 \mathrm{D}$ density-response function takes the Drude form $(1 / 2 \pi)\left(v_{F}^{2 D} q / \omega\right)^{2}$, and one finds

$$
\alpha=\sqrt{2 z_{d}}
$$

which is the result first obtained by Chaplik in his study of charge-carrier crystallization in low-density inversion layers ${ }^{73}$

If the $2 \mathrm{D}$ sheet is located inside the $3 \mathrm{D}$ substrate $(z \leq 0)$, however, the long-wavelength limit of the effective 2D dielectric function of Eq. (34) has no zeros at low energies $\left(\omega<\omega_{s}\right)$, due to a complete screening at these energies of electron-electron interactions within the $2 \mathrm{D}$ sheet. This result has suggested over the years that acoustic plasmons should only exist in the case of spatially separated plasmas, as pointed out by Das Sarma and Madhukar 40

Nevertheless, Silkin et al 44 have shown that metal surfaces where a partially occupied quasi-2D surface-state band coexists in the same region of space with the underlying 3D continuum do support a well-defined acoustic surface plasmon, which could not be explained within the local model described above. Furthermore, it has been demonstrated that it is a combination of the nonlocality of the 3D dynamical screening and the spill out of the $3 \mathrm{D}$ electron density into the vacuum which allows the formation of $2 \mathrm{D}$ electron-density acoustic oscillations at metal surfaces, since these oscilations would otherwise be completely screened by the surrounding $3 \mathrm{D}$ substrate. 46

\section{B. Full calculation}

In order to achieve a full description of the dynamical response of real metal surfaces, we first consider a one-dimensional single-particle potential that describes the main features of the surface band structure ${ }^{74.75} \mathrm{We}$ then calculate the eigenfunctions and eigenvalues of the corresponding hamiltonian, and we evaluate the dynamical density-response function $\chi^{0}\left(z, z^{\prime} ; q, \omega\right)$. Finally, we solve an integral equation to obtain the RPA interacting density-response function $\chi\left(z, z^{\prime} ; q, \omega\right)$. From the knowledge of this function, which describes bulk and surface states on the same footing, one can obtain within linearresponse theory the electron density induced by an external perturbation $\phi^{e x t}(z ; q, \omega)$ :

$$
\delta n(z ; q, \omega)=\int d z^{\prime} \chi\left(z, z^{\prime} ; q, \omega\right) \phi^{e x t}\left(z^{\prime} ; q, \omega\right),
$$

and the collective excitations created by an external potential of the form

$$
\phi^{e x t}(z ; q, \omega)=-(2 \pi / q) \mathrm{e}^{q z}
$$

can then be traced to the peaks of the imaginary part of the surface-response function 51

$$
\operatorname{Im}[g(q, \omega)]=\int d z \mathrm{e}^{q z} \operatorname{Im}[\delta n(z ; q, \omega)] .
$$

In the bottom panel of Fig. [10] we show the result that we have obtained for the unperturbed electron density of the (0001) surface of Be. We see bulk states, whose total density extends to the interior of the solid at $z<0$, and 

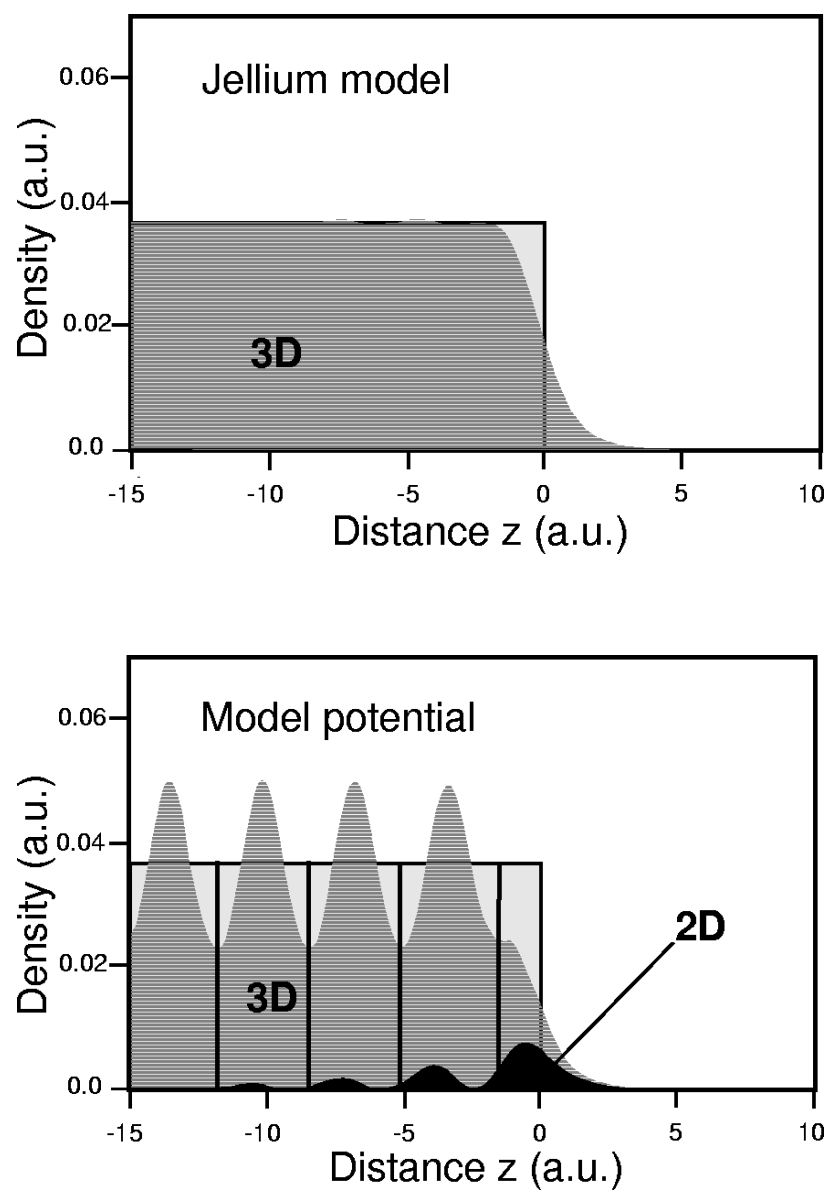

FIG. 10: Unperturbed electron density of the (0001) surface of Be (dark grey shaded areas), as obtained from a self-consistent jellium density-functional calculation (top panel) and from the use of a one-dimensional potential that describes the main features of the surface band structure (bottom panel). The light grey shaded areas represent the neutralizing uniform positive background. The vertical lines and black area in the bottom panel, which are absent in the top panel, represent the atomic positions of the solid and the unperturbed density of occupied surface states, respectively. The crystal edge $(z=0)$ is chosen to be located half a lattice spacing beyond the last atomic layer, and $z<0$ corresponds to the interior of the solid.

surface states which are largely localized near the surface. For comparison, we have also carried out a self-consistent jellium density-functional calculation of the electron density (top panel of Fig. 10), by simply replacing the ions of the solid by a fixed uniform positive background. Surface states are absent in this model.

RPA calculations of the imaginary part of the electron density $\delta n(z ; q, \omega)$ induced in a $\mathrm{Be}(0001)$ surface by the external potential of Eq. (38) were reported in Ref. 44 for $q=0.05 a_{0}^{-1}\left(a_{0}\right.$ is the Bohr radius, $a_{0}=0.529 \AA$ ) and a wide range of frequencies. It was demonstrated that this quantity exhibits two distinct special features, where $\operatorname{Im}[\delta n(q, \omega)]$ is maximum near the surface. The first feature occurs near the surface-plasmon frequency of valence $\left(2 s^{2}\right)$ electrons in $\mathrm{Be}\left(\hbar \omega_{s}=12.8 \mathrm{eV}\right)$. The second feature occurs at $\omega=0.6 \mathrm{eV}$, corresponding to a new low-energy acoustic collective oscilation, which had been overlooked over the years.

In Fig. 11] we show by black lines our full RPA calculation of $\operatorname{Im}[\delta n(z ; q, \omega)]$ at $q=0.05 a_{0}^{-1}$ and the frequencies $\omega=12.8 \mathrm{eV}$ (top panel) and $\omega=0.6 \mathrm{eV}$ (bottom panel) at which surface collective oscillations occur. At the conventional surface-plasmon frequency $\omega=12.8 \mathrm{eV}$ we have also carried out a jellium density-functional calculation for a semi-infinite free-electron gas, which we have represented by a grey line in the top panel of Fig. 11. A comparison of our band-structure and jellium calculations (black and grey lines) indicates that the conventional surface plasmon is reasonably well described within a jellium model of the surface, although Friedel oscillations in the interior of the solid are considerably more damped in the presence of the actual band structure of the solid.

At the acoustic surface-plasmon energy, which for $q=$ $0.05 a_{0}^{-1}$ is $\omega=0.6 \mathrm{eV}$, a quasi-2D surface-state band in the presence of a $3 \mathrm{D}$ substrate yields the new feature displayed in the bottom panel of Fig. 11] Also shown in this figure (shaded area) is the probability density of the partially occupied Shockley surface state, clearly indicating that the low-energy collective excitation at $\omega=0.6 \mathrm{eV}$ originates from this $2 \mathrm{D}$ surface-state band. Such a $2 \mathrm{D}$ electron gas alone would only support a plasmon that for $q=0.05 a_{0}^{-1}$ has energy $\omega_{2 D}=2.7 \mathrm{eV}$, well above the low-energy excitation that is visible in the bottom panel of Fig. 11] and it is only the combination of the strongly localized 2D surface-state band with 3D bulk states which allows the formation of this new mode.

Fig. 12 shows the imaginary part of the surfaceresponse function $g(q, \omega)$ of $\mathrm{Be}(0001)$, as obtained from Eq. (39) for increasing values of $q$. This figure clearly shows that the excitation spectra is dominated at low energies by a well-defined acoustic peak with linear dispersion, the sound velocity being at long wavelengths very close to the 2D Fermi velocity $v_{F}^{2 D}$ (see Table II).

In the bottom panel of Fig. 13 we show the energy of the acosutic surface plasmon in $\mathrm{Be}(0001)$ versus the wave number $q$ (thick solid line), as derived from the maxima of our calculated surface-loss function $\operatorname{Im}[g(q, \omega)]$ of Fig. 12 The plasmon energy of electrons in an isolated 2D electron gas would exhibit at long wavelengths a square-root dispersion with the wave number $q$ [see Eq. (19)]. However, the combination of a 2D surfacestate band with the underlying $3 \mathrm{D}$ system yields a new distinct mode whose energy lies just above the upper edge $\omega_{u}^{2 D}=v_{F}^{2 D} q+q^{2} / 2$ of the continuum of $2 \mathrm{D}$ e-h pair excitations (shaded area), where momentum and energy conservation allows e-h pairs to be created within the $2 \mathrm{D}$ electron gas.

For a well-defined acoustic surface plasmon to occur, it must exist for wave vectors $\mathbf{q}$ and energies $\omega$ where decay cannot occur by exciting e-h pairs in the medium. Electron-hole pairs can be excited either within the 2D 

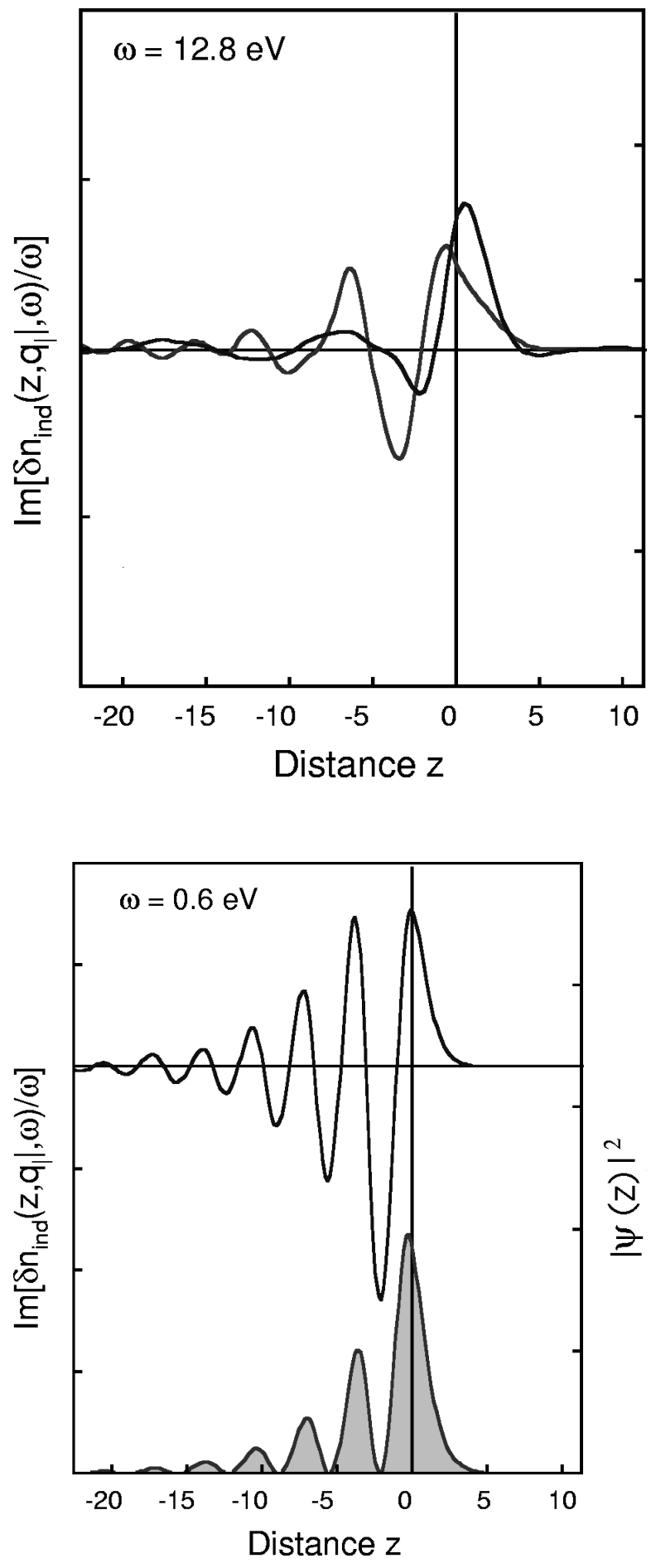

FIG. 11: RPA calculation of the imaginary part of the electron density induced in the (0001) surface of Be (black lines), as obtained from Eqs. (37) and (38) for a wave vector of magnitude $q=0.05 a_{0}^{-1}$, as a function of the $z$ coordinate normal to the surface. The frequency has been chosen to be $\omega=0.6 \mathrm{eV}$ (bottom panel) and $\omega=12.8 \mathrm{eV}$ (top panel). The grey line in the top panel represents the corresponding jellium density-functional calculation for a semi-infinite freeelectron gas. The probability density of the partially occupied Shockley surface state is represented in the bottom panel by a shaded area. As in Fig. [10 the crystal edge $(z=0)$ is chosen to be located half a lattice spacing beyond the last atomic layer, and $z<0$ corresponds to the interior of the solid.

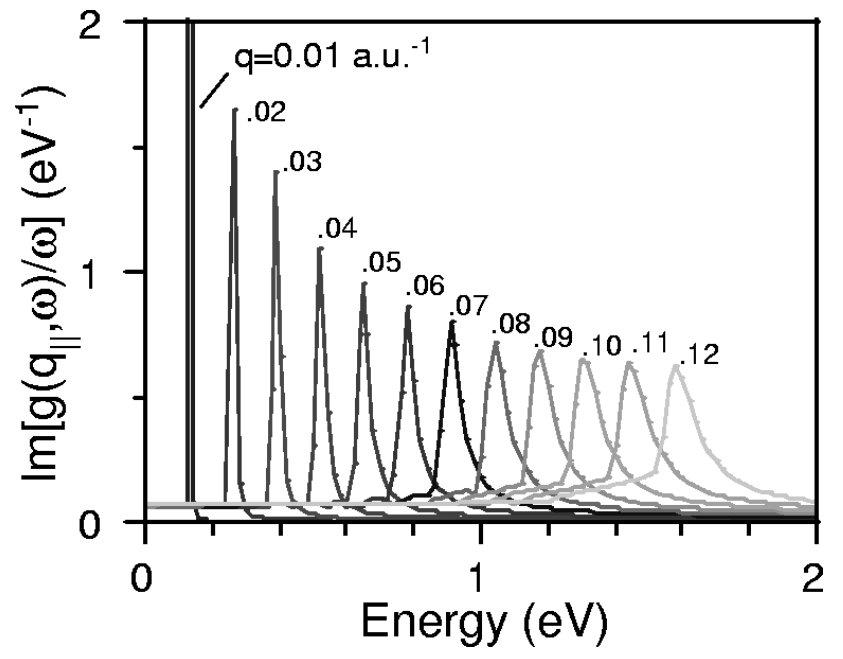

FIG. 12: Energy-loss function $\operatorname{Im}[g(q, \omega)] / \omega$ of $\operatorname{Be}(0001)$ versus the energy $\omega$, as obtained from Eq. (39) for various values of the wave number $q$, in units of the inverse Bohr radius $a_{0}^{-1}$. The peaks are dictated by the corresponding poles of the surface response function $g(q, \omega)$. In the long-wavelenth $(q \rightarrow 0)$ limit, $g(q, \omega)$ is simply the total electron density induced by the potential of Eq. (38).

surface-state band, or within the 3D continuum of bulk states, or by promoting an electron from an occupied bulk state to an unoccupied 2D surface state. These three mechanisms for the production of e-h pairs at low energies are illustrated in Fig. 13. (i) Since the energy of acoustic surface plasmons always lies just above the upper edge $\omega_{u}^{2 D}$ of the 2D e-h pair continuum (shaded area in the bottom panel of Fig. [13), they cannot possibly decay by creating e-h pairs within the $2 \mathrm{D}$ band. (ii) The 3D Fermi velocity $v_{F}^{3 D}$ is typically larger than the Fermi velocity $v_{F}^{2 D}$ of the $2 \mathrm{D}$ surface-state band. This means that acoustic surface plasmons can decay by exciting e-h pairs within the 3D continuum of bulk states. However, at the low energies involved the probability for this pro-

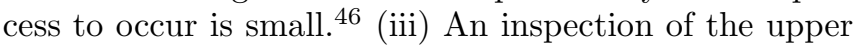
panel of Fig. 13] shows that, due to the presence of the band gap, for optical $(q=0)$ transitions to occur from an occupied 3D bulk state to an unoccupied 2D surface state a minimum energy is required, which decreases as the momentum transfer $q$ increases.

The region of momentum space where transitions from $3 \mathrm{D}$ to $2 \mathrm{D}$ states cannot occur corresponds to the area below the thin solid line in the bottom panel of Fig. 13. This figure shows that at long wavelengths with $q<0.06 a_{0}^{-1}$ acoustic surface plasmons can decay by neither exciting 2D e-h pairs nor exciting 3D-2D e-h pairs, which results in a very well-defined collective excitation (see Fig. 12). At shorter wavelengths $\left(q>0.06 a_{0}^{-1}\right)$, the promotion of electrons from occupied 3D bulk states to unoccupied 2D surface states becomes possible and the corresponding plasmon peak broadenes considerably. 

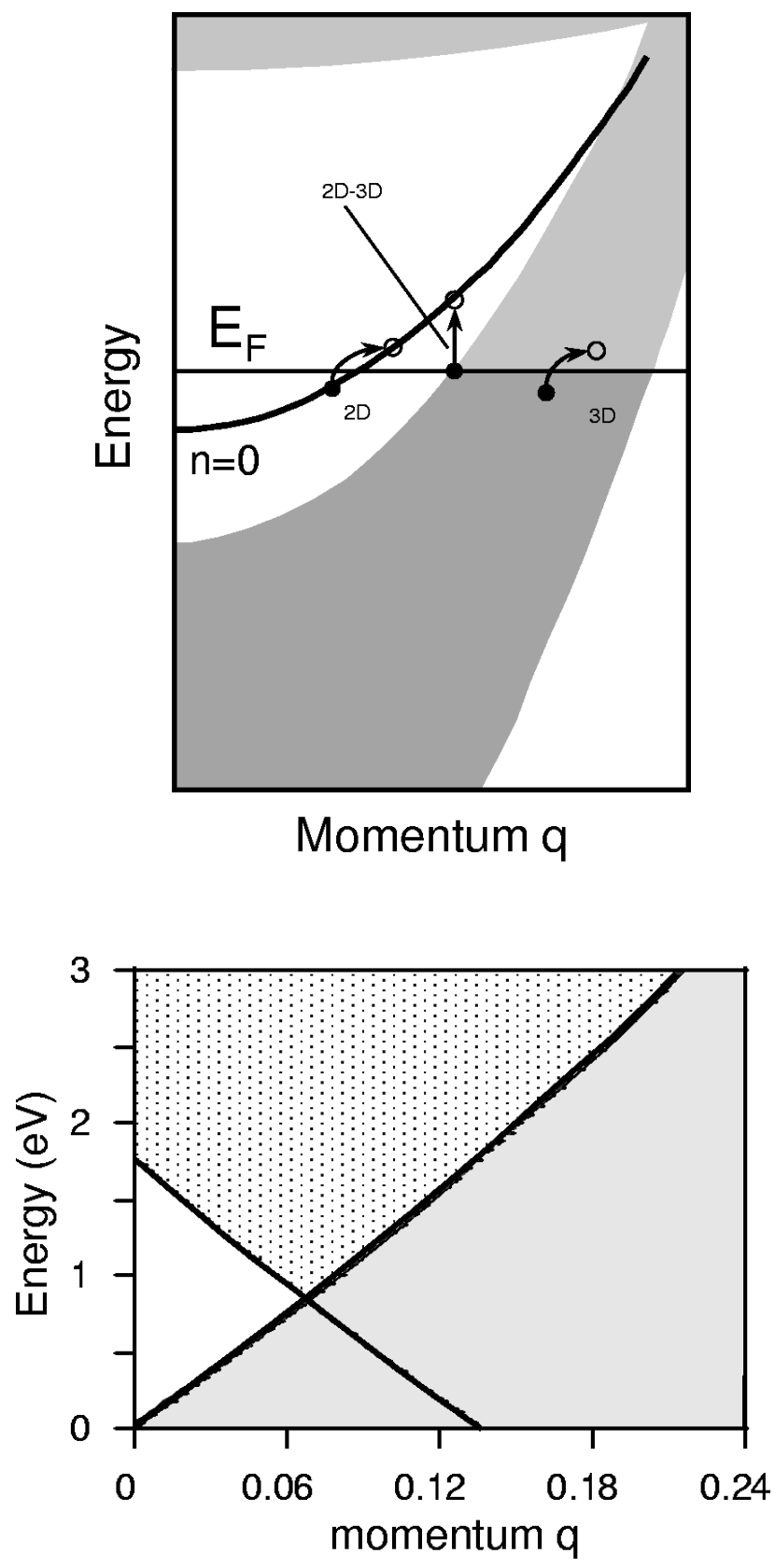

FIG. 13: Top panel: Schematic representation of the projection of the bulk bands onto a solid surface that supports a partially occupied Shockley surface-state band. The energy of occupied and unoccupied states is displayed as a function of the momentum parallel to the surface. The solid line represents a Shockley surface state. Dark and light shaded areas represent occupied and unoccupied bulk states, respectively. The white area represents the band gap. Electron-hole pair excitations are represented by arrows, depending on whether they correspond to transitions within the surface-state band (2D), transitions within the bulk bands (3D), or transitions from bulk to surface states (2D-3D). Bottom panel: Dispersion of the acoustic surface collective excitation of $\mathrm{Be}(0001)$, as derived from the peaks that are visible in Fig. 12 (thick solid line). This curve stays just over the upper edge $\omega_{u}^{2 D}=v_{F}^{2 D} q+q^{2} / 2$ of the $2 \mathrm{D}$ e-h pair continuum (shaded area). Momentum and energy conservation prevent $2 \mathrm{D}$ e-h pairs from being produced for energies above $\omega_{u}^{2 D}$. The area below the thin solid line represents the region of momentum space where transitions from $3 \mathrm{D}$ to $2 \mathrm{D}$ states cannot occur. Well-defined acoustic plasmons are expected to occur at wave vectors with magnitude smaller than $q \sim 0.06 a_{0}^{-1}$.
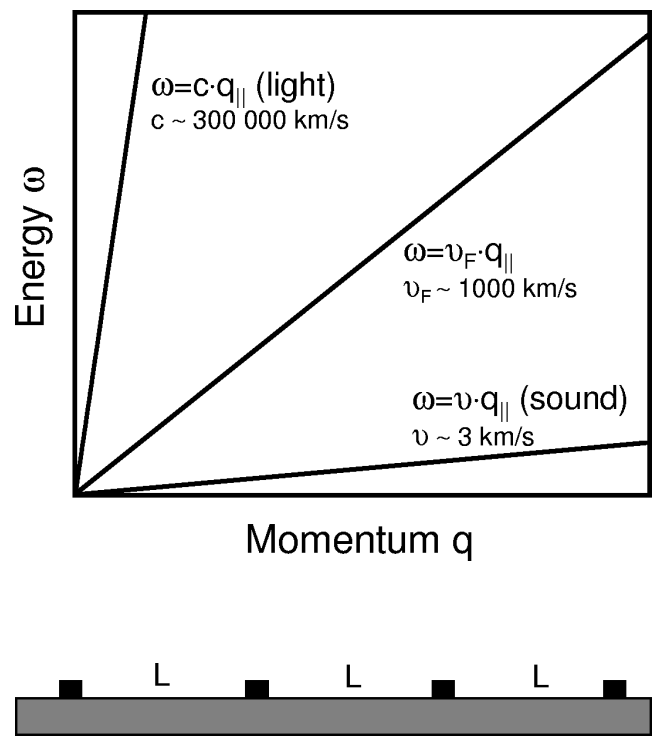

FIG. 14: Top panel: Schematic (out of scale) representation of typical energy dispersions of acoustic surface plasmons, acoustic phonons, and free-space electromagnetic radiation. In the actual scale, the dispersion lines of free light and acoustic phonons should be very close to the vertical and horizontal axes, respectively. Bottom panel: Periodic grating of constant $L$. The grating periodic structure can provide the impigning free electromagnetic radiation with additional momentum $2 \pi / L$.

\section{Excitation of acoustic surface plasmons}

We close this paper by discussing whether acoustic surface plasmons can be observed. As in the case of conventional surface plasmons, acoustic surface plasmons should be expected to be excited by either electrons or light. Here we focus on a possible mechanism that would lead to the excitation of acoustic surface plasmons by light in, e.g., vicinal surfaces with high indices.

The top panel of Fig. 14 exhibits the energy dispersion of acoustic surface plasmons at low wave vectors. Also shown in this figure are the light line $\omega=c q$ and, for comparison, a typical energy dispersion of acoustic phonons. The sound velocity of acoustic surface plasmons, which is close to the Fermi velocity of the 2D surface-state band, is typically a few orders of magnitude larger than the sound velocity of acoustic phonons in metals but still about two orders of magnitude smaller than the velocity of light.

The acoustic surface plasmon dispersion curve is well below the dispersion curve of free-space electromagnetic radiation. Hence, there is, in principle, no way that incident light can provide an ideal surface with the correct amount of momentum and energy for the excitation of an acoustic surface plasmon. As in the case of conventional surface plasmons, however, a periodic corrugation or grating in the metal surface should be able to provide the missing momentum.

If light hits a surface with a periodic corrugation, the 
grating (see bottom panel of Fig. 14) can provide the impigning free electromagnetic waves with additional momentum arising from the grating periodic structure. If free electromagnetic radiation hits the grating at an angle $\theta$, its wave vector along the grating surface has magnitude

$$
q=\frac{\omega}{c} \sin \theta \pm \frac{2 \pi}{L} n
$$

where $L$ represents the grating constant, and $n=1,2, \ldots$. Hence, the linear (nearly vertical) dispersion relation of free light changes into a set of parallel straight lines, which can match the acoustic-plasmon dispersion relation.

For a well-defined acoustic surface plasmon in $\mathrm{Be}(0001)$ to be observed, the wave number $q$ needs to be smaller than $q \sim 0.06 a_{0}^{-1}$. For $q=0.05 a_{0}^{-1}$, Eq. (40) with $n=1$ yields a grating constant $L=66 \AA$. Acoustic surface plas- mons of energy $\omega \sim 0.6 \mathrm{eV}$ could be excited in this way. Although a grating period of a few nanometers sounds unrealistic with present technology, the possible control of vicinal surfaces with high indices could provide appropriate grating periods in the near future. Alternatively, acoustic surface plasmons could be observed with the use of high-resolution electron energy-loss spectroscopy (EELS) under grazing incidence.

\section{Acknowledgments}

Partial support by the University of the Basque Country, the Basque Unibertsitate eta Ikerketa Saila, the Spanish Ministerio de Educación y Cultura, and the Max Planck Research Award Funds is gratefully acknowledged.
1 D. Pines and D. Bohm, Phys. Rev. 85, 338 (1952).

2 D. Pines, Rev. Mod. Phys. 28, 184 (1956).

3 The valence electron density $n$ is usually characterized by the density parameter $r_{s}=(3 / 4 \pi n)^{3} / a_{0}$ ( $a_{0}$ is the Bohr radius, $\left.a_{0}=0.529 \AA\right)$. In metals $\left(2<r_{s}<6\right)$, plasmons have energies in the range $5 \mathrm{eV}<\omega_{p}<20 \mathrm{eV}$ and frequencies that lie, therefore, in the optical regime.

4 D. Gabor, Phil. Mag. 1, 1 (1956).

${ }^{5}$ R. H. Ritchie, Phys. Rev. 106, 874 (1957).

${ }^{6}$ E. A. Stern and R. A. Ferrell, Phys. Rev. 120, 130 (1960).

7 C. J. Powell and J. B. Swan, Phys. Rev. 115, 869 (1959); 116, 81 (1959).

8 P. J. Feibelman, Surf. Sci. 27, 438 (1971).

9 R. H. Ritchie, Phys. Lett. A 38, 189 (1972).

10 R. Ray amd G. D. Mahan, Phys. Lett. A 42, 306 (1972).

11 A. Sunjic, G. Toulouse, and A. A. Lucas, Solid State Commun. 11, 1629 (1972).

12 J. Schmit and A. A. Lucas, Solid State Commun. 11, 415 (1972).

13 P. M. Echenique and J. B. Pendry, J. Phys. C: Solid State Phys. 8 (1975).

14 P. M. Echenique, R. H. Ritchie, N. Barberan, and J. Inkson, Phys. Rev. B 23, 6486 (1981).

15 P. J. Feibelman, Prog. Surf. Sci. 12, 287 (1982).

16 R. H. Ritchie, Prog. Theor. Phys. 29, 607 (1963).

17 R. H. Ritchie and A. L. Marusak, Surf. Sci. 4, 234 (1966).

18 D. E. Beck, Phys. Rev. B 4, 1555 (1971). Phys.

19 P. Hohenber and W. Kohn, Phys. Rev. 136 B864 (1964); W. Kohn and L. J. Sham, Phys. Rev. A1133 (1965).

${ }^{20}$ K.-D. Tsuei, E. W. Plummer, A. Liebsch, K. Kempa, and P. Bakshi, Phys. Rev. Lett. 64, 44 (1989).

21 W. Plummer, K.-D. Tsuei, and B.-O. Kim, Nucl. Instrum. Methods B 96, 448 (1995).

22 A. J. Benett, Phys. Rev. B 1, 203 (1970).

23 Throughout this paper, long wavelengths are associated with low wave vectors.

${ }^{24}$ R. H. Ritchie and H. B. Eldridge, Phys. Rev. 126, 1935 (1962).

25 Y.-Y. Teng and E. A. Stern, Phys. Rev. Lett. 19, 511 (1967).
26 A. Otto, Z. Phys. 216, 398 (1968).

27 E. Kretschmann and H. Raether, Z. Naturforsch. A 23, 2135 (1968).

28 J. R. Sambles, G. W. Bradbery, and F. Z. Yang, Contemp. Phys. 32, 173 (1991).

29 R. Berndt, J. K. Gimzewski, and P. Johansson, Phys. Rev. 67, 3796 (1991).

30 M. J. Shea and R. N. Compton, Phys. Rev. B 47, 9967 (1993).

31 M. Rocca, Surf. Sci. Rep. 22, 1 (1995).

32 B. Rothenhausler and W. Knoll, Nature 332, 615 (1988).

33 S. C. Schuster, R. V. Swanson, L. A. Alex, R. B. Bourret, and M. I. Simon, Nature 365, 343 (1993).

34 J. B. Pendry, Science 265, 1687 (1999).

35 T. W. Ebbesen, H. J. Lezec, H. F. Ghaemi, T. Thio, and P. A. Wolff, Nature 391, 667 (1998).

36 H. J. Lezec, A. Degiron, E. Devaux, R. A. Linke, L. MartinMoreno, F. J. García-Vidal, and T. W. Ebbesen, Science 297, 820 (2002)

37 W. L. Barnes, A. Dereux, and T. W. Ebbesen, Nature 424, 824 (2003).

38 D. Pines, Can. J. Phys. 34, 1379 (1956).

39 N. H. March and M. P. Tosi, Adv. Phys. 44, 299 (1995).

40 S. Das Sarma and A. Madhukar, Phys. Rev. B 23, 805 (1981).

41 D. Olego, A. Pinczuk, A. C. Gossard, and W. Wiegmann, Phys. Rev. B 32, 1921 (1985).

42 J. Ruvalds, Phys. Rev. B 35, 8869 (1987); Nature 328, 299 (1987).

43 A. Bill, H. Morawitz, and V. Z. Kresin, Phys. Rev. B 66, 100501 (2002).

${ }^{44}$ V. M. Silkin, A. García-Lekue, J. M. Pitarke, E. V. Chulkov, E. Zaremba, and P. M. Echenique, Eurphys. Lett. 66, 260 (2004).

45 The sound velocity of this acoustic mode is, however, close to the Fermi velocity of the 2D surface-state band, which is typically a few orders of magnitude larger than the sound velocity of acoustic phonons in metals but still about two orders of magnitude smaller than the velocity of light.

46 J. M. Pitarke, V. U. Nazarov, V. M. Silkin, E. V. Chulkov, 
E. Zaremba, and P. M. Echenique (unpublished).

47 This approximation is valid for heavy charged particles, e.g., ions, and also for swift electrons moving with nonrelativistic velocities that are large compared to the velocity of target electrons. In the case of electrons, $Z_{1}=-1$.

${ }^{48}$ F. Flores and F. García Moliner, Introduction to the Theory of Solid Surfaces (Cambridge University Press, Cambridge, 1979).

49 A. L. Fetter and J. D. Wallecka, Quantum Theory of ManyParticle Systems (McGraw-Hill, New York, 1964).

50 J. Lindhard, K. Dan. Vidensk. Selsk. Mat. Fys. Medd. 28, 8 (1954).

51 See, e.g., A. Liebsch, Electronic Excitations at Metal Surfaces (Plenum Press, New York, 1997).

52 R. A. Ferrell, Phys. Rev. 111, 1214 (1958).

${ }^{53}$ F. Stern, Phys. Rev. Lett. 18, 546 (1967); T. Ando, A. B. Fowler, and F. Stern, Rev. Mod. Phys. 54, 437 (1982).

${ }^{54}$ S. J. Allen jr., D. C. Tsui, and R. A. Logan, Phys. Rev. Lett. 38, 980 (1977).

55 T. Nagao, T. Heldebrandt, M. Henzler, and S. Hasegava, Phys. Rev. Lett. 86, 5747 (2001).

56 M. Abramowitz and I. A. Stegun, Handbokk of Mathematical Functions (Dover, New York, 1965).

57 R. Nuñez, P. M. Echenique, and R. H. Ritchie, J. Phys. C: Solid State Phys. 13, 4229 (1980).

${ }^{58}$ H. Ibach and D. L. Mills, Electron Energy Loss Spectroscopy and Surface Vibrations (Academic, New York, 1982).

59 K.-D. Tsuei, E. W. Plummer, A. Liebsch, E. Pehlke, K.
Kempa, and P. Bakshi, Surf. Sci. 247, 302 (1991).

${ }^{60}$ P. T. Sprunger, G. M. Watson, and E. W. Plummer, Surf. Sci. 269, 551 (1992).

61 E. Zaremba and W. Kohn, Phys. Rev. B 13, 2270 (1976).

62 P. Apell and C. Holmberg, Solid State Commun. 49, 693 (1984).

63 J. Tarriba and W. L. Mochan, Phys. Rev. 46, 12902 (1992).

64 P. J. Feibelman, Surf. Sci. 282, 129 (1993).

65 A. Liebsch, Phys. Rev. Lett. 71, 145 (1993).

66 S. P. Apell, P. M. Echenique, and R. H. Ritchie, Ultramicroscopy 65, 53 (1995).

67 A. Ronveaux, A. Moussiaux, and A. A. Lucas, Can. J. Phys. 55, 1407 (1977).

68 H. W. Kroto, J. R. Heath, S. C. O'Brien, R. F. Curl, and R. E. Smalley, Nature 318, 162 (1985).

${ }^{69}$ S. Ijima, Nature 354, 56 (1991).

70 D. Östling, P. Apell, and A. Rosén, Europhys. Lett. 21, 539 (1993).

71 H. Raether, Surface Plasmons, Springer Tracks in Modern Physics, 111 (New York, Springer, 1988).

72 J. E. Inglesfield, Rep. Prog. Phys. 45, 223 (1982).

73 A. V. Chaplik, Zh. Eksp. Teor. Diz. 62, 746 (1972) (Sov. Phys. JETP, 35, 395 (1972)).

74 E. V. Chulkov, V. M. Silkin, and P. M. Echenique, Surf. Sci. 437, 330 (1999).

75 P. M. Echenique, J. M. Pitarke, E. V. Chulkov, and A. Rubio, Chem. Phys. 251, 1 (2000). 